\title{
ON THE POSITIVITY OF TRACE CLASS OPERATORS
}

\author{
ELENA CORDERO, MAURICE DE GOSSON, AND FABIO NICOLA
}

\begin{abstract}
The characterization of positivity properties of Weyl operators is a notoriously difficult problem, and not much progress has been made since the pioneering work of Kastler, Loupias, and Miracle-Sole (KLM). In this paper we begin by reviewing and giving simpler proofs of some known results for trace-class Weyl operators; the latter play an essential role in quantum mechanics. We then apply time-frequency analysis techniques to prove a phase space version of the KLM condition; the main tools are Gabor frames and the Wigner formalism. Finally, discrete approximations of the KLM condition, which are tractable numerically, are provided.
\end{abstract}

\section{INTRODUCTION}

The characterization of positivity properties for trace class operators on $L^{2}\left(\mathbb{R}^{n}\right)$ is an important topic, not only because it is an interesting mathematical problem which still is largely open, but also because of its potential applications to quantum mechanics and even cosmology. It is a notoriously difficult part of functional analysis which has been tackled by many authors but there have been few decisive advances since the pioneering work of Kastler 13 and Loupias and Miracle-Sole [15, 16]; see however Dias and Prata [4]. While some partial results have been obtained in connection with the study of quantum density operators [18, 19, 20, 21, 22] when the operators under consideration are expressed using the Weyl correspondence, very little is known about them when they are given in terms of more general correspondences (in [27] Srinivas and Wolf give such a condition, but the necessity statement is false as already noted by Mourgues et al. [17]). It seems in fact that the field, which was quite active in the late 1980s hasn't much evolved since; the open questions remain open.

We shall tackle the problem using techniques which come from both quantum mechanics and time-frequency analysis. The phase space

2010 Mathematics Subject Classification. 46E35, 35S05, 81S30, 42C15.

Key words and phrases. Wigner transform, trace class operator, positive operator, Weyl symbol, Gabor frames. 
representation mainly employed is the $\eta$-cross-Wigner transform; for $\eta \in \mathbb{R} \backslash\{0\}$, this is defined by

$$
W_{\eta}(\psi, \phi)(z)=\left(\frac{1}{2 \pi \eta}\right)^{n} \int_{\mathbb{R}^{n}} e^{-\frac{i}{\eta} p \cdot y} \psi\left(x+\frac{1}{2} y\right) \overline{\phi\left(x-\frac{1}{2} y\right)} d y,
$$

for $\psi, \phi \in L^{2}\left(\mathbb{R}^{n}\right)$. When $\eta=\hbar>0$, ( $\hbar$ the Planck constant $h$ divided by $2 \pi)$ we recapture the standard cross-Wigner function $W_{\hbar}(\psi, \phi)$, simply denoted by $W(\psi, \phi)$. Setting $W_{\eta}(\psi, \psi)=W_{\eta} \psi$ and $\lambda=\eta / \hbar$, we have

$$
W_{\eta} \psi(x, p)=|\lambda|^{-n} W \psi\left(x, \lambda^{-1} p\right) .
$$

In particular, a change of $\eta$ into $-\eta$ yields

$$
W_{\eta} \psi=(-1)^{n} W_{-\eta} \bar{\psi}
$$

Given a symbol $a \in \mathcal{S}^{\prime}\left(\mathbb{R}^{2 n}\right)$ (the space of tempered distribution), the Weyl pseudodifferential operator $\widehat{A}_{\eta}^{\mathrm{W}}=\mathrm{Op}_{\eta}^{\mathrm{W}}(a)$ is weakly defined by

$$
\left\langle\widehat{A}_{\eta}^{\mathrm{W}} \psi, \bar{\phi}\right\rangle=\left\langle a, W_{\eta}(\psi, \phi)\right\rangle
$$

for all $\psi, \phi$ in the Schwartz class $\mathcal{S}\left(\mathbb{R}^{n}\right)$ (Observe that $W_{\eta}(\psi, \phi) \in$ $\left.\mathcal{S}\left(\mathbb{R}^{2 n}\right)\right)$. The function $a$ is called the $\eta$-Weyl symbol of $\widehat{A}_{\eta}^{\mathrm{W}}$.

Consider now a trace-class operator $\widehat{A}$ on $L^{2}\left(\mathbb{R}^{n}\right)$ (see the definition in the subsequent Section 3). Then there exists an orthonormal basis $\left(\psi_{j}\right)$ for $L^{2}\left(\mathbb{R}^{n}\right)$ and a sequence $\left(\alpha_{j}\right) \in \ell^{1}$ such that $\widehat{A}$ can be written as

$$
\widehat{A}=\sum_{j} \alpha_{j} \widehat{\Pi}_{j}
$$

whit absolute convergence in $B\left(L^{2}\left(\mathbb{R}^{n}\right)\right)$; here $\widehat{\Pi}_{j}$ is the rank-one orthogonal projector of $L^{2}\left(\mathbb{R}^{n}\right)$ onto the one-dimensional subspace $\mathbb{C} \psi_{j}$ generated by $\psi_{j}$ (cf. Lemma 10). It turns out that, under the additional assumption $\widehat{A}$ to be self-adjoint, that $\widehat{A}$ can be represented as a $\eta$-Weyl operator with corresponding symbol

$$
a=(2 \pi \eta)^{n} \sum_{j} \alpha_{j} W_{\eta} \psi_{j} \in L^{2}\left(\mathbb{R}^{2 n}\right) \cap L^{\infty}\left(\mathbb{R}^{2 n}\right)
$$

(see Proposition 11).

When $\widehat{A}$ is positive semidefinite and has trace equal to one, it is called a density operator (or density matrix, or stochastic, operator in quantum mechanics); it is usually denoted by $\widehat{\rho}$. If the Weyl symbol of $\widehat{\rho}$ is $a$, the function $\rho=(2 \pi \eta)^{-n} a$ is called the Wigner distribution of 
$\widehat{\rho}$ in the quantum mechanical literature. Given a trace class operator $\widehat{A}$ (positive or not), the function

$$
\rho=\sum_{j} \alpha_{j} W_{\eta} \psi_{j}
$$

is called the $\eta$-Wigner distribution of $\widehat{A}$. (Observe that $\rho \in L^{2}\left(\mathbb{R}^{2 n}\right)$ ).

We will henceforth assume that all the concerned operators are selfadjoint and of trace class and denote them by $\widehat{\rho}$; such operators can always be written as

$$
\widehat{\rho}=\sum_{j} \alpha_{j} \widehat{\Pi}_{j}=(2 \pi \eta)^{n} \mathrm{Op}_{\eta}^{\mathrm{W}}(\rho)
$$

the real function $\rho$ being given by formula (5). We are going to determine explicit necessary and sufficient conditions on $\rho$ ensuring the positivity of $\widehat{\rho}$. To this goal, we will use the reduced symplectic Fourier transform $F_{\diamond}$, defined for $a \in \mathcal{S}\left(\mathbb{R}^{2 n}\right)$ by

$$
a_{\diamond}(z)=F_{\diamond} a(z)=\int_{\mathbb{R}^{2 n}} e^{i \sigma\left(z, z^{\prime}\right)} a\left(z^{\prime}\right) d z^{\prime}
$$

with $\sigma$ being the standard symplectic form. For $\eta \in \mathbb{R} \backslash\{0\}$, recall the symplectic $\eta$-Fourier transform

$$
a_{\sigma, \eta}(z)=F_{\sigma, \eta} a(z)=\left(\frac{1}{2 \pi \eta}\right)^{n} \int_{\mathbb{R}^{2 n}} e^{-\frac{i}{\eta} \sigma\left(z, z^{\prime}\right)} a\left(z^{\prime}\right) d z^{\prime} .
$$

Obviously $F_{\diamond}$ is related to the symplectic $\eta$-Fourier transform (8) by the formula

$$
a_{\diamond}(z)(z)=(2 \pi \eta)^{n} a_{\sigma, \eta}(-\eta z) .
$$

With the notation (7) Bochner's theorem [3, 14] on Fourier transforms of probability measures can be restated in the following way:

Proposition 1 (Bochner). A real function $\rho \in L^{1}\left(\mathbb{R}^{2 n}\right)$ is a probability density if and only if $\rho_{\diamond}$ is continuous, $\rho_{\diamond}(0)=1$, and for all $z_{1}, \ldots, z_{N} \in$ $\mathbb{R}^{2 n}$ the $N \times N$ matrix $\Lambda$ whose entries are the complex numbers $\rho_{\diamond}\left(z_{j}-\right.$ $\left.z_{k}\right)$ is positive semidefinite:

$$
\Lambda=\left(\rho_{\diamond}\left(z_{j}-z_{k}\right)\right)_{1 \leq j, k \leq N} \geq 0 .
$$

When condition (10) is satisfied one says that $\rho_{\diamond}$ is of positive type. The notion of $\eta$-positivity, due to Kastler [13], generalizes this notion:

Definition 2. Let $a \in L^{1}\left(\mathbb{R}^{2 n}\right)$ and $\eta \in \mathbb{R} \backslash\{0\}$; we say that $a_{\diamond}$ is of $\eta$-positive type if for every integer $N$ the $N \times N$ matrix $\Lambda_{(N)}$ with entries

$$
\Lambda_{j k}=e^{-\frac{i \eta}{2} \sigma\left(z_{j}, z_{k}\right)} a_{\diamond}\left(z_{j}-z_{k}\right)
$$


is positive semidefinite for all choices of $\left(z_{1}, z_{2}, \ldots, z_{N}\right) \in\left(\mathbb{R}^{2 n}\right)^{N}$ :

$$
\Lambda_{(N)}=\left(\Lambda_{j k}\right)_{1 \leq j, k \leq N} \geq 0 .
$$

The condition (11) is equivalent to the polynomial inequalities

$$
\sum_{1 \leq j, k \leq N} \zeta_{j} \overline{\zeta_{k}} e^{-\frac{i \eta}{2} \sigma\left(z_{j}, z_{k}\right)} a_{\diamond}\left(z_{j}-z_{k}\right) \geq 0
$$

for all $N \in \mathbb{N}, \zeta_{j}, \zeta_{k} \in \mathbb{C}$, and $z_{j}, z_{k} \in \mathbb{R}^{2 n}$.

It is easy to see that this implies $a_{\diamond}(-z)=\overline{a_{\diamond}(z)}$ and therefore $a$ is real-valued.

Remark 3. If a is of $\eta$-positive type then it is also of $(-\eta)$-positive type. This follows from the fact that the matrix $\left(\overline{\Lambda_{j k}}\right)_{1 \leq j, k \leq N}$ is still positive semidefinite and taking into account the equality $\overline{a_{\diamond}(z)}=a_{\diamond}(-z)$.

We first present a result originally due to Kastler [13], and Loupias and Miracle-Sole [15, 16] (the "KLM conditions"), who use the theory of $C^{*}$-algebras; also see Parthasarathy [23, 24] and Parthasarathy and Schmidt [25]. The proof we give is simpler and is partially based on the discussions in [19, 21, 28].

Theorem 4 (The KLM conditions). Let $\eta \in R \backslash\{0\}$ and let $\widehat{A}=$ $\mathrm{Op}_{\eta}^{\mathrm{W}}(a)$ be a self-adjoint trace-class operator on $L^{2}\left(\mathbb{R}^{n}\right)$ with symbol $a \in L^{1}\left(\mathbb{R}^{2 n}\right)$. We have $\widehat{A} \geq 0$ if and only if the conditions below hold:

(i) $a_{\diamond}$ is continuous;

(ii) $a_{\diamond}$ is of $\eta$-positive type.

The KLM conditions are difficult to use in practice since they involve the simultaneous verification of an uncountable set of conditions. We are going to prove that they can be replaced with a countable set of conditions in phase space. The key idea from time-frequency analysis is to use Gabor frames.

Definition 5. Given a lattice $\Lambda$ in $\mathbb{R}^{2 n}$ and a non-zero function $g \in$ $L^{2}\left(\mathbb{R}^{n}\right)$, the system

$$
\mathcal{G}(g, \Lambda)=\left\{T(\lambda) g(x)=e^{i\left(\lambda_{2} x-\frac{1}{2} \lambda_{1} \lambda_{2}\right)} g\left(x-\lambda_{1}\right), \lambda=\left(\lambda_{1}, \lambda_{2}\right) \in \Lambda\right\}
$$

is called a Gabor frame or Weyl-Heisenberg frame if it is a frame for $L^{2}\left(\mathbb{R}^{n}\right)$, that is there exist constants $0<A \leq B$ such that

$$
A\|f\|_{2}^{2} \leq \sum_{z \in \Lambda}|\langle f, T(\lambda) g\rangle|^{2} \leq B\|f\|_{2}^{2}, \quad \forall f \in L^{2}\left(\mathbb{R}^{n}\right) .
$$

Hence, the $L^{2}$-norm of the function $f$ is equivalent to the $\ell^{2}$ norm of the sequence of its coefficients $\left\{\left\langle f, T_{1 /(2 \pi)}(\lambda) g\right\rangle\right\}_{\lambda \in \Lambda}$ (cf. Section 2 
for more details). Consider a Gabor frame $\mathcal{G}(\phi, \Lambda)$ for $L^{2}\left(\mathbb{R}^{n}\right)$, with window $\phi \in L^{2}\left(\mathbb{R}^{n}\right)$ and lattice $\Lambda \in \mathbb{R}^{n}$. Let $a \in \mathcal{S}^{\prime}\left(\mathbb{R}^{2 n}\right)$ be a symbol and denote by $a_{\lambda, \mu}$ its "twisted" Gabor coefficient with respect to the Gabor system $\mathcal{G}\left(W_{\eta} \phi, \Lambda \times \Lambda\right)$, defined for $\lambda, \mu \in \Lambda \times \Lambda$ by

$$
a_{\lambda, \mu}=\int_{\mathbb{R}^{2 n}} e^{-\frac{i}{\eta} \sigma(z, \lambda-\mu)} a(z) W_{\eta} \phi\left(z-\frac{1}{2}(\lambda+\mu)\right) d z,
$$

where $W_{\eta} \psi=W_{\eta}(\psi, \psi)$ is the $\eta$-Wigner transform of $\psi$.

Our main result characterizes the positivity of Hilbert-Schmidt operators (and hence of trace class operators). It reads as follows:

Theorem 6. Let $a \in L^{2}\left(\mathbb{R}^{n}\right)$ be real-valued and $\widehat{A}_{\eta}=\mathrm{Op}_{\eta}^{\mathrm{W}}(a)$.

(i) We have $\widehat{A}_{\eta} \geq 0$ if and only if for every integer $N \geq 0$ the matrix $M_{(N)}$ with entries

$$
M_{\lambda, \mu}=e^{-\frac{i}{2 \eta} \sigma(\lambda, \mu)} a_{\lambda, \mu} \quad, \quad|\lambda|,|\mu| \leq N
$$

is positive semidefinite.

(ii) One obtains an equivalent statement replacing the matrix $M_{(N)}$ with the matrix $M_{(N)}^{\prime}$ where

$$
M_{\lambda, \mu}^{\prime}=W_{\eta}\left(a,\left(W_{\eta} \phi\right)^{\vee}\right)\left(\frac{1}{4}(\lambda+\mu), \frac{1}{2} J(\mu-\lambda)\right)
$$

with $\left(W_{\eta} \phi\right)^{\vee}(z)=W_{\eta} \phi(-z)$.

The conditions in Theorem 6 only involve a countable set of matrices, as opposed to the KLM ones. In addition, they are well-organized because the matrix of size $N$ is a submatrix of that of size $N+1$.

The KLM conditions can be recaptured by an averaging procedure from the ones in Theorem 6. To show this claim, we make use of another well-known time-frequency representation: the short-time Fourier transform (STFT). Precisely, for a given function $g \in \mathcal{S}\left(\mathbb{R}^{n}\right) \backslash\{0\}$ (called window), the STFT $V_{g} f$ of a distribution $f \in \mathcal{S}^{\prime}\left(\mathbb{R}^{n}\right)$ is defined by

$$
V_{g} f(x, p)=\int_{\mathbb{R}^{n}} e^{-i p \cdot y} f(y) \overline{g(y-x)} d y, \quad(x, p) \in \mathbb{R}^{2 n}
$$

Let $\phi_{0}(x)=(\pi \eta)^{-n / 4} e^{-|x|^{2} / 2 \eta}$ be the standard Gaussian and $\phi_{\nu}=$ $T(\nu) \phi_{0}, \nu \in \mathbb{R}^{2 n}$. We shall consider the STFT $V_{W \phi_{\nu}} a$, with window given by the Wigner function $W \phi_{\nu}$ and symbol $a$. Then we establish the following connection: 
Theorem 7. Let $a \in L^{1}\left(\mathbb{R}^{2 n}\right)$ and $\lambda, \mu \in \mathbb{R}^{2 n}$. We set

$$
\begin{aligned}
M_{\lambda, \mu}^{(K L M)} & =e^{-\frac{i}{2 \eta} \sigma(\lambda, \mu)} a_{\sigma, \eta}(\lambda-\mu) \\
M_{\lambda, \mu}^{\phi_{\nu}} & =e^{-\frac{i}{2 \eta} \sigma(\lambda, \mu)} V_{W \phi_{\nu}} a\left(\frac{1}{2}(\lambda+\mu), J(\mu-\lambda)\right) .
\end{aligned}
$$

We have

$$
M_{\lambda, \mu}^{(K L M)}=(2 \pi \eta)^{-n} \int_{\mathbb{R}^{2 n}} M_{\lambda, \mu}^{\phi_{\nu}} d \nu
$$

If the symbol $a \in L^{1}\left(\mathbb{R}^{n}\right) \cap L^{2}\left(\mathbb{R}^{n}\right)$ and choosing the lattice $\Lambda$ such that $\mathcal{G}\left(\phi_{0}, \Lambda\right)$ is a Gabor frame for $L^{2}\left(\mathbb{R}^{n}\right)$, we obtain the following consequence: If the matrix $\left(M_{\lambda, \mu}^{\phi_{0}}\right)_{\lambda, \mu \in \Lambda,|\lambda|,|\mu| \leq N}$ is positive semidefinite for every $N$, then so is the matrix $\left(M_{\lambda, \mu}^{(K L M)}\right)_{\lambda, \mu \in \Lambda,|\lambda|,|\mu| \leq N}$ (cf. Corollary 24).

Finally, if the symbol $a$ is as before and and $\widehat{A}_{\eta}=\mathrm{Op}_{\eta}^{\mathrm{W}}(a) \geq 0$, then for every finite subset $S \subset \mathbb{R}^{2 n}$ the matrix $\left(M_{\lambda, \mu}^{(K L M)}\right)_{\lambda, \mu \in S}$ is positive semidefinite. That is, the KLM conditions hold (see Corollary 25).

The paper is organized as follows:

- In Section 2 we briefly recall the main definitions and properties of the Wigner-Weyl-Moyal formalism.

- In Section 3 we discuss the notion of positivity for trace class operators; we also prove a continuous version of the positivity theorem using the machinery of Hilbert-Schmidt operators.

- In Section 4 we characterize positivity using the Kastler-LoupiasMiracle-Sole (KLM) conditions of which we give a simple proof. We give a complete description of trace class operators with Gaussian Weyl symbols using methods which simplify and put on a rigorous footing older results found in the physical literature.

- In Section 5 we show that the KLM conditions, which form an uncountable set of conditions can be replaced with a set of countable conditions involving the Wigner function. We thereafter study the notion of "almost positivity" which is an useful approximation of the notion of positivity which can be easily implemented numerically.

Notation 8. We denote by $z=(x, p)$ the generic element of $\mathbb{R}^{2 n} \equiv$ $\mathbb{R}^{n} \times \mathbb{R}^{n}$. Equipping $\mathbb{R}^{2 n}$ with the symplectic form $\sigma=\sum_{j} d p_{j} \wedge d x_{j}$ we denote by $\operatorname{Sp}(n)$ the symplectic group of $\left(\mathbb{R}^{2 n}, \sigma\right)$ and by $\operatorname{Mp}(n)$ the corresponding metaplectic group. $J=\left(\begin{array}{cc}0_{n \times n} & I_{n \times n} \\ -I_{n \times n} & 0_{n \times n}\end{array}\right)$ is the standard 
symplectic matrix, and we have $\sigma\left(z, z^{\prime}\right)=J z \cdot z^{\prime}$. The $L^{2}$-scalar product is given by

$$
(\psi \mid \phi)_{L^{2}}=\int_{\mathbb{R}^{n}} \psi(x) \overline{\phi(x)} d x .
$$

The distributional pairing between $\psi \in \mathcal{S}^{\prime}\left(\mathbb{R}^{m}\right)$ and $\phi \in \mathcal{S}\left(\mathbb{R}^{m}\right)$ is denoted by $\langle\psi, \phi\rangle$ regardless of the dimension $m$.

For $A, B \in \mathrm{GL}(m)$, we use the notation $A \backsim B$ to denote the equality of two square matrices $A, B$ of same size $m \times m$ up to conjugation: $A \sim B$ if and only if there exists $C \in \mathrm{GL}(m)$ such that $A=C^{-1} B C$.

\section{Weyl Operators and Gabor frames}

2.1. The Weyl-Wigner formalism. In what follows $\eta$ denotes a real parameter different from zero.

Given a symbol $a \in \mathcal{S}^{\prime}\left(\mathbb{R}^{2 n}\right)$ the Weyl pseudodifferential operator $\widehat{A}_{\eta}^{\mathrm{W}}=\mathrm{Op}_{\eta}^{\mathrm{W}}(a)$ is defined in (4), whereas the $\eta$-cross-Wigner transform $W_{\eta}(\psi, \phi)$ is recalled in (11).

The operator $T_{\eta}(z)$ is Heisenberg's $\eta$-displacement operator

$$
T_{\eta}\left(z_{0}\right) \psi(x)=e^{\frac{i}{\eta}\left(p_{0} x-\frac{1}{2} p_{0} x_{0}\right)} \psi\left(x-x_{0}\right)
$$

(see [6, 7]). The $\eta$-cross-ambiguity transform is defined by

$$
\operatorname{Amb}_{\eta}(\psi, \phi)(z)=\left(\frac{1}{2 \pi \eta}\right)^{n}\left(\psi \mid T_{\eta}(z) \phi\right)_{L^{2}} ;
$$

we have [5, 7] the relation

$$
\operatorname{Amb}_{\eta}(\psi, \phi)=F_{\sigma, \eta} W_{\eta}(\psi, \phi),
$$

where $F_{\sigma, \eta}$ is the symplectic $\eta$-Fourier transform already recalled in (8). The functions $W_{\eta} \psi=W_{\eta}(\psi, \psi)$ and $\operatorname{Amb}_{\eta} \psi=\operatorname{Amb}_{\eta}(\psi, \psi)$ are called, respectively, the $\eta$-Wigner and $\eta$-ambiguity transforms. The explicit expression of the $\eta$-Wigner transform is already given in (1), whereas the $\eta$-ambiguity transform is defined by

$$
\operatorname{Amb}_{\eta}(\psi, \phi)(z)=\left(\frac{1}{2 \pi \eta}\right)^{n} \int_{\mathbb{R}^{n}} e^{-\frac{i}{\eta} p \cdot y} \psi\left(y+\frac{1}{2} x\right) \overline{\phi\left(y-\frac{1}{2} x\right)} d y .
$$

Let $\widehat{A}_{\eta}^{\mathrm{W}}=\mathrm{Op}_{\eta}^{\mathrm{W}}(a)$ and $\widehat{B}_{\eta}^{\mathrm{W}}=\mathrm{Op}_{\eta}^{\mathrm{W}}(b)$ and assume that $\widehat{A}_{\eta}^{\mathrm{W}} \widehat{B}_{\eta}^{\mathrm{W}}$ is defined on some subspace of $L^{2}\left(\mathbb{R}^{n}\right)$; then the twisted symbol $c_{\sigma, \eta}$ of $\widehat{C}_{\eta}^{\mathrm{W}}=\widehat{A}_{\eta}^{\mathrm{W}} \widehat{B}_{\eta}^{\mathrm{W}}$ is given by the "twisted convolution" [5, [7] $c_{\sigma, \eta}=$ $a_{\sigma, \eta} *_{\eta} b_{\sigma, \eta}$ defined by

$$
\left(a_{\sigma, \eta} \star_{\eta} b_{\sigma, \eta}\right)(z)=\left(\frac{1}{2 \pi \eta}\right)^{n} \int_{\mathbb{R}^{2 n}} e^{\frac{i}{2 \eta} \sigma\left(z, z^{\prime}\right)} a_{\sigma, \eta}\left(z-z^{\prime}\right) b_{\sigma, \eta}\left(z^{\prime}\right) d z^{\prime} .
$$

Alternatively, the symbol $c$ is given by the "twisted product" $c=a \times_{\hbar} b$ where 


$$
\left(a \times_{\eta} b\right)(z)=\left(\frac{1}{4 \pi \eta}\right)^{2 n} \int_{\mathbb{R}^{2 n}} e^{\frac{i}{2 \eta} \sigma\left(z^{\prime}, z^{\prime \prime}\right)} a\left(z+\frac{1}{2} z^{\prime}\right) b\left(z-\frac{1}{2} z^{\prime \prime}\right) d z^{\prime} d z^{\prime \prime} .
$$

An important property of the $\eta$-Wigner transform is that it satisfies the "marginal properties"

$$
\int_{\mathbb{R}^{n}} W_{\eta} \psi(z) d x=\left|F_{\eta} \psi(p)\right|^{2}, \quad \int_{\mathbb{R}^{n}} W_{\eta} \psi(z) d p=|\psi(x)|^{2},
$$

the first for every function $\psi \in L^{1}\left(\mathbb{R}^{n}\right) \cap L^{2}\left(\mathbb{R}^{n}\right)$, the second for every function $\psi \in L^{2}\left(\mathbb{R}^{n}\right)$ such that $\hat{\psi} \in L^{1}\left(\mathbb{R}^{n}\right)$; here

$$
F_{\eta} \psi(p)=\left(\frac{1}{2 \pi|\eta|}\right)^{n / 2} \int_{\mathbb{R}^{n}} e^{-\frac{i}{\eta} p x} \psi(x) d x
$$

is the $\eta$-Fourier transform (see [5, 8]). Notice that $F_{\eta} \psi$ and $F_{-\eta} \psi$ are related by the trivial formula

$$
F_{-\eta} \psi=(-1)^{n} \overline{F_{\eta} \bar{\psi}}
$$

It follows that $F_{\eta}$ extends into a topological unitary automorphism of $L^{2}\left(\mathbb{R}^{n}\right)$ for all values of $\eta \neq 0$.

An important equality satisfied by the $\eta$-Wigner function is Moyal's identity 1 :

Lemma 9. Let $(\psi, \phi) \in L^{2}\left(\mathbb{R}^{n}\right) \times L^{2}\left(\mathbb{R}^{n}\right)$ and $\eta \in \mathbb{R} \backslash\{0\}$. The function $W_{\eta} \psi$ is real and we have

$$
\left\|W_{\eta} \psi\right\|_{L^{2}\left(\mathbb{R}^{2 n}\right)}^{2}=\int_{\mathbb{R}^{2 n}} W_{\eta} \psi(z) W_{\eta} \phi(z) d z=\left(\frac{1}{2 \pi|\eta|}\right)^{n}|(\psi \mid \phi)|^{2} .
$$

In particular

$$
\int_{\mathbb{R}^{2 n}} W_{\eta} \psi(z)^{2} d z=\left(\frac{1}{2 \pi|\eta|}\right)^{n}\|\psi\|^{4}
$$

Proof. It is a standard result [5, 12, that (29) holds for all $\eta>0$. The case $\eta<0$ follows using formula (3) .

In Section 5 we will use some concepts from time-frequency analysis. We recall here the most important issues.

A Gabor frame $\mathcal{G}(\phi, \Lambda)$ is defined in Definition (5). This implies that any function $f \in L^{2}\left(\mathbb{R}^{n}\right)$ can be represented as

$$
f=\sum_{\lambda \in \Lambda} c_{\lambda} T(\lambda) g
$$

with unconditional convergence in $L^{2}\left(\mathbb{R}^{n}\right)$ and with suitable coefficients $\left(c_{\lambda}\right)_{\lambda} \in \ell^{2}(\Lambda)$.

\footnotetext{
${ }^{1}$ It is sometimes also called the "orthogonality relation" for the Wigner function.
} 
A time-frequency representation closely related to the Wigner function is the short-time Fourier transform (STFT), whose definition is in formula (17). Using this representation, we can define the Sjöstrand class or modulation space $M_{v_{s}}^{\infty, 1}[11,26]$ in terms of the decay of the STFS as follows. For $s \geq 0$, consider the weight function $v_{s}(z)=\langle z\rangle^{s}=$ $\left(1+|z|^{2}\right)^{s / 2}, z \in \mathbb{R}^{2 n}$, then

$M_{v_{s}}^{\infty, 1}\left(\mathbb{R}^{n}\right)=\left\{f \in \mathcal{S}^{\prime}\left(\mathbb{R}^{n}\right):\|f\|_{M_{v_{s}}^{\infty, 1}}:=\int_{\mathbb{R}^{n}} \sup _{x \in \mathbb{R}^{n}}\left|V_{g} f(x, p)\right| v_{s}(x, p) d p<\infty\right\}$.

It can be shown that $\|f\|_{M_{v_{s}}^{\infty, 1}}$ is a norm on $M_{v_{s}}^{\infty, 1}\left(\mathbb{R}^{n}\right)$, independent of the window function $g \in \mathcal{S}\left(\mathbb{R}^{n}\right)$ (different windows yield equivalent norms). Moreover $M_{v_{s}}^{\infty, 1}\left(\mathbb{R}^{n}\right)$ is a Banach space. For $s=0$ we simply write $M^{\infty, 1}\left(\mathbb{R}^{n}\right)$ in place of $M_{v_{s}}^{\infty, 1}\left(\mathbb{R}^{n}\right)$.

Generally, by measuring the decay of the STFT by means of the mixed-normed spaces $L_{v_{s}}^{p, q}\left(\mathbb{R}^{2 n}\right)$, one can define a scale of Banach spaces known as modulation spaces. Here we will make use only of the socalled Feichtinger's algebra (unweighted case $s=0$ )

$$
M^{1}\left(\mathbb{R}^{n}\right)=\left\{f \in \mathcal{S}^{\prime}\left(\mathbb{R}^{2 n}\right):\|f\|_{M^{1}}=\left\|V_{g} f\right\|_{L^{1}\left(\mathbb{R}^{2 n}\right)}<\infty\right\} .
$$

Notice that in Section 5 we will work with spaces of symbols, hence the dimension $n$ of the space is replaced by $2 n$.

\section{The Positivity of Trace-Class Weyl Operators}

Trace class operators play an essential role in quantum mechanics. A positive semidefinite self-adjoint operator with unit trace is called a density operator (or density matrix in the physical literature). Density operators represent (and are usually identified with) the mixed quantum states corresponding to statistical mixtures of quantum pure states.

3.1. Trace class operators. A bounded linear operator $\widehat{A}$ on $L^{2}\left(\mathbb{R}^{n}\right)$ is of trace class if for one (and hence every) orthonormal basis $\left(\psi_{j}\right)_{j}$ of $L^{2}\left(\mathbb{R}^{n}\right)$ its modulus $|\widehat{A}|=\left(\widehat{A}^{*} \widehat{A}\right)^{1 / 2}$ satisfies

$$
\sum_{j}\left(|\widehat{A}| \psi_{j} \mid \psi_{j}\right)_{L^{2}}<\infty
$$

the trace of $\widehat{A}$ is then, by definition, given by the absolutely convergent series $\operatorname{Tr}(\widehat{A})=\sum_{j}\left(\widehat{A} \psi_{j} \mid \psi_{j}\right)_{L^{2}}$ whose value is independent of the choice of the orthonormal basis $\left(\psi_{j}\right)_{j}$.

Trace class operators form a two-side ideal $\mathcal{L}_{1}\left(L^{2}\left(\mathbb{R}^{n}\right)\right)$ in the algebra $\mathcal{L}\left(L^{2}\left(\mathbb{R}^{n}\right)\right)$ of all bounded linear operators on $L^{2}\left(\mathbb{R}^{n}\right)$. 
An operator $\widehat{A} \in \mathcal{L}\left(L^{2}\left(\mathbb{R}^{n}\right)\right)$ is a Hilbert-Schmidt operator if and only if there exists an orthonormal basis $\left(\psi_{j}\right)$ such that

$$
\sum_{j}\left(\widehat{A} \psi_{j} \mid \widehat{A} \psi_{j}\right)_{L^{2}}<\infty
$$

Hilbert-Schmidt operators form a two-sided ideal $\mathcal{L}_{2}\left(L^{2}\left(\mathbb{R}^{n}\right)\right)$ in $\mathcal{L}\left(L^{2}\left(\mathbb{R}^{n}\right)\right)$ and we have $\mathcal{L}_{1}\left(L^{2}\left(\mathbb{R}^{n}\right)\right) \subset \mathcal{L}_{2}\left(L^{2}\left(\mathbb{R}^{n}\right)\right)$. A trace class operator can always be written (non uniquely) as the product of two Hilbert-Schmidt operators (and is hence compact). In particular, a positive (and hence self-adjoint) trace-class operator can always be written in the form $\widehat{A}=\widehat{B}^{*} \widehat{B}$ where $\widehat{B}$ is a Hilbert-Schmidt operator. One proves ([2], $\S 22.4$, also [6, 7]) using the spectral theorem for compact operators that for every trace-class operator on $L^{2}\left(\mathbb{R}^{n}\right)$ there exists a sequence $\left(\alpha_{j}\right)_{j} \in \ell^{1}(\mathbb{N})$ and orthonormal bases $\left(\psi_{j}\right)_{j}$ and $\left(\phi_{j}\right)_{j}$ of $L^{2}\left(\mathbb{R}^{n}\right)$ (indexed by the same set) such that for $\psi \in L^{2}\left(\mathbb{R}^{n}\right)$

$$
\widehat{A} \psi=\sum_{j} \alpha_{j}\left(\psi \mid \psi_{j}\right)_{L^{2}} \phi_{j}
$$

conversely the formula above defines a trace-class operator on $L^{2}\left(\mathbb{R}^{n}\right)$. Observe that the series in (32) is absolutely convergent in $L^{2}\left(\mathbb{R}^{n}\right)$ (hence unconditionally convergent), since

$\sum_{j}\left\|\alpha_{j}\left(\psi \mid \psi_{j}\right)_{L^{2}} \phi_{j}\right\|=\sum_{j}\left|\alpha_{j}\right|\left|\left(\psi \mid \psi_{j}\right)_{L^{2}}\right|\left\|\phi_{j}\right\| \leq \sum_{j}\left|\alpha_{j}\right|\|\psi\|\left\|\psi_{j}\right\|=\sum_{j}\left|\alpha_{j}\right|\|\psi\|$.

One verifies that the adjoint $\widehat{A}^{*}$ (which is also of trace class) is given by

$$
\widehat{A}^{*} \psi=\sum_{j} \overline{\alpha_{j}}\left(\psi \mid \phi_{j}\right)_{L^{2}} \psi_{j}
$$

where the series is absolutely convergent in $L^{2}\left(\mathbb{R}^{n}\right)$.

The following issue is an easy consequence of the spectral theorem for compact self-adjoint operators.

Lemma 10. Let $\widehat{A}$ be a trace-class operator on $L^{2}\left(\mathbb{R}^{n}\right)$.

(i) If $\widehat{A}$ is self-adjoint there exists a real sequence $\left(\alpha_{j}\right)_{j} \in \ell^{1}(\mathbb{N})$ and an orthonormal basis $\left(\psi_{j}\right)_{j}$ of $L^{2}\left(\mathbb{R}^{n}\right)$ such that

$$
\widehat{A} \psi=\sum_{j} \alpha_{j}\left(\psi \mid \psi_{j}\right)_{L^{2}} \psi_{j}
$$

for every $\psi \in L^{2}\left(\mathbb{R}^{n}\right)$, with absolute convergence in $L^{2}\left(\mathbb{R}^{n}\right)$;

(ii) if $\widehat{A} \geq 0$ then (34) holds with $\alpha_{j} \geq 0$ for all $j$. 
Formula (34) can be rewritten for short as

$$
\widehat{A}=\sum_{j} \alpha_{j} \widehat{\Pi}_{j}
$$

where $\widehat{\Pi}_{j}$ is a rank-one projector, namely the orthogonal projection operator of $L^{2}\left(\mathbb{R}^{n}\right)$ onto the one-dimensional subspace $\mathbb{C} \psi_{j}$ generated by $\psi_{j}$.

Notice that the series (35) is absolutely convergent in $B\left(L^{2}\left(\mathbb{R}^{n}\right)\right)$. Indeed, $\left\|\widehat{\Pi}_{j}\right\|_{B\left(L^{2}\right)}=1$ for every $j$ and

$$
\sum_{j}\left\|\alpha_{j} \widehat{\Pi}_{j}\right\|_{B\left(L^{2}\right)} \leq \sum_{j}\left|\alpha_{j}\right|<\infty .
$$

Proposition 11. Let $\widehat{A}$ be a self-adjoint trace-class operator on $L^{2}\left(\mathbb{R}^{n}\right)$ as in (34) and $\eta>0$.

(i) The $\eta$-Weyl symbol a of $\widehat{A}$ is given by

$$
a=(2 \pi \eta)^{n} \sum_{j} \alpha_{j} W_{\eta} \psi_{j}
$$

where the series converges absolutely in $L^{2}\left(\mathbb{R}^{2 n}\right)$;

(ii) The twisted symbol $a_{\sigma, \eta}$ is given by

$$
a_{\sigma, \eta}=(2 \pi \eta)^{n} \sum_{j} \alpha_{j} \operatorname{Amb}_{\eta} \psi_{j} .
$$

with absolute convergence in $L^{2}\left(\mathbb{R}^{2 n}\right)$.

(iii) In particular, the symbols $a$ and $a_{\sigma, \eta}$ are in $L^{2}\left(\mathbb{R}^{2 n}\right) \cap L^{\infty}\left(\mathbb{R}^{2 n}\right)$.

Proof. The distributional kernel of the orthogonal projection $\widehat{\Pi}_{j}$ is $K_{j}=\psi_{j} \otimes \overline{\psi_{j}}$ hence the Weyl symbol $a_{j}$ of $\widehat{\Pi}_{j}$ is given by the usual formula

$$
a_{j}(z)=\int_{\mathbb{R}^{n}} e^{-\frac{i}{\eta} p y} K_{j}\left(x+\frac{1}{2} y, x-\frac{1}{2} y\right) d y=(2 \pi \eta)^{n} W_{\eta} \psi_{j}(z) .
$$

the series (35) being absolutely convergent in $B\left(L^{2}\left(\mathbb{R}^{n}\right)\right)$. Moyal's identity

$$
\left\|\alpha_{j} W_{\eta} \psi_{j}\right\|_{2}=\left|\alpha_{j}\right|\left(\frac{1}{2 \pi \eta}\right)^{\frac{n}{2}}\left\|\psi_{j}\right\|_{2}^{2}=\left(\frac{1}{2 \pi \eta}\right)^{\frac{n}{2}}\left|\alpha_{j}\right|
$$

and the assumption $\left(\alpha_{j}\right)_{j} \in \ell^{1}(\mathbb{N})$ guarantee that the series in (36) is absolutely convergent in $L^{2}\left(\mathbb{R}^{2 n}\right)$ and we infer that the symbol $a$ is in $L^{2}\left(\mathbb{R}^{2 n}\right)$. Similarly, by Hölder's inequality,

$$
\left|W_{\eta} \psi_{j}(z)\right| \leq \frac{2^{2 n}}{(2 \pi \eta)^{n}}\left\|\psi_{j}\right\|_{2}^{2}
$$


for all $z \in \mathbb{R}^{2 n}$ so that

$$
\left\|\alpha_{j} W_{\eta} \psi_{j}\right\|_{\infty} \leq\left(\frac{2}{\pi \eta}\right)^{n}\left|\alpha_{j}\right|
$$

and the series in (36) is absolutely convergent in $L^{\infty}\left(\mathbb{R}^{2 n}\right)$, too. This proves our claim (iii) for the symbol $a$. Formula (37) follows since $W_{\eta} \psi_{j}$ and $\mathrm{Amb}_{\eta} \psi_{j}$ are symplectic $\eta$-Fourier transforms of each other. Claim (iii) for $a_{\sigma, \eta}$ is obtained in a similar way.

Remark 12. From Proposition (11), the functions $a$ and $a_{\sigma, \eta}$ are in $L^{2}\left(\mathbb{R}^{2 n}\right) \cap L^{\infty}\left(\mathbb{R}^{2 n}\right)$. Notice that in general the symbols $a$ and $a_{\sigma, \eta}$ are not in $L^{1}\left(\mathbb{R}^{2 n}\right)$. For example, choose $\widehat{A}=\widehat{\Pi}_{0}$, with $\widehat{\Pi}_{0}$ the orthogonal projection onto a vector $\psi_{0} \in L^{2}\left(\mathbb{R}^{n}\right) \backslash\left(L^{1}\left(\mathbb{R}^{n}\right) \cup \mathcal{F} L^{1}\left(\mathbb{R}^{n}\right)\right)$.

Recall that if $\widehat{A}$ is positive semidefinite and has trace equal to one, it is called a density operator and denoted by $\widehat{\rho}$. We will from now on assume that all the concerned operators are self-adjoint and of trace class, recalling from (6) that they can be written as

$$
\widehat{\rho}=\sum_{j} \alpha_{j} \widehat{\Pi}_{j}=(2 \pi \eta)^{n} \mathrm{Op}_{\eta}^{\mathrm{W}}(\rho)
$$

the real function $\rho$ being given by formula (5). We are going to determine explicit necessary and sufficient conditions on $\rho$ ensuring the positivity of $\widehat{\rho}$. Let us first note the following result which shows the sensitivity of density operators to changes in the value of $\hbar$.

Let us now address the following question: for given $\psi \in L^{2}\left(\mathbb{R}^{n}\right)$, can we find $\phi$ such that $W_{\eta} \phi=W \psi$ for $\eta \neq \hbar$ ? The answer is negative:

Proposition 13. Let $\psi \in L^{1}\left(\mathbb{R}^{n}\right) \cap L^{2}\left(\mathbb{R}^{n}\right) \backslash\{0\}$ and $\eta \in \mathbb{R} \backslash\{0\}$, $\hbar>0$.

(i) There does not exist any $\phi \in L^{1}\left(\mathbb{R}^{n}\right) \cap L^{2}\left(\mathbb{R}^{n}\right)$ such that $W_{\eta} \phi=$ $W \psi$ if $|\eta| \neq \hbar$.

(ii) Assume that there exist orthonormal systems $\left(\psi_{j}\right)_{j \in \mathbb{N}},\left(\phi_{j}\right)_{j \in \mathbb{N}}$ of $L^{2}\left(\mathbb{R}^{n}\right)$ and nonnegative sequences $\alpha=\left(\alpha_{j}\right)_{j \in \mathbb{N}}, \beta=\left(\beta_{j}\right)_{j \in \mathbb{N}} \in \ell^{1}(\mathbb{N})$ such that

$$
\sum_{j} \alpha_{j} W_{\eta} \psi_{j}=\sum_{j} \beta_{j} W \phi_{j}
$$

Then we must have

$$
\hbar^{n}\|\alpha\|_{\ell^{2}}^{2}=|\eta|^{n}\|\beta\|_{\ell^{2}}^{2} .
$$

Proof. Observe that the series in (38) are absolutely convergent in $L^{2}\left(\mathbb{R}^{2 n}\right)$. (i) Assume that $W_{\eta} \phi=W \psi$; then, using the first marginal 
property (26),

$$
\left|F_{\eta} \phi(p)\right|^{2}=\int_{\mathbb{R}^{n}} W_{\eta} \phi(x, p) d x=\int_{\mathbb{R}^{n}} W \psi(x, p) d x=\left|F_{\hbar} \psi(p)\right|^{2}
$$

hence $\phi$ and $\psi$ must have the same $L^{2}$-norm: $\|\phi\|=\|\psi\|$ in view of Parseval's equality. On the other hand, using the Moyal identity (29) for, respectively, $W \psi$ and $W_{\eta} \phi$, the equality $W \psi=W_{\eta} \phi$ implies that

$$
\begin{aligned}
\int_{\mathbb{R}^{2 n}} W \psi(z)^{2} d z & =\left(\frac{1}{2 \pi \hbar}\right)^{n}\|\psi\|^{4} \\
\int_{\mathbb{R}^{2 n}} W_{\eta} \phi(z)^{2} d z & =\left(\frac{1}{2 \pi|\eta|}\right)^{n}\|\phi\|^{4}
\end{aligned}
$$

hence we must have $|\eta|=\hbar$.

(ii) Squaring both sides of (38) and integrating over $\mathbb{R}^{2 n}$ we get, using again Moyal's identity and the orthonormality of the vectors $\psi_{j}$ and $\phi_{j}$,

$$
\frac{1}{(2 \pi|\eta|)^{n}} \sum_{j} \alpha_{j}^{2}=\frac{1}{(2 \pi \hbar)^{n}} \sum_{j} \beta_{j}^{2}
$$

hence our claim.

Remark 14. Assume in particular that $\beta_{1}=1$ and $\beta_{j}=0$ for $j \geq$ 2. Then (ii) tells us that if $\sum_{j} \alpha_{j} W_{\eta} \psi_{j}=W \phi$ then we must have $\hbar^{n}\|\alpha\|_{\ell^{2}}^{2}=|\eta|^{n}$. Assume that $\sum_{j} \alpha_{j}=1$; then $\|\alpha\|_{\ell^{2}}^{2} \leq 1$ hence we must have $|\eta| \leq \hbar$.

\section{Positive Trace Class Operators}

4.1. A general positivity result for trace class operators. We are now going to give an integral description of the positivity of a trace class operator on $L^{2}\left(\mathbb{R}^{n}\right)$ of which Theorem 16 can be viewed as a discretized version.

Let us begin by stating a general result:

Lemma 15. Let $\widehat{A}=\mathrm{Op}_{\eta}^{\mathrm{W}}(a)$ be a trace-class operator on $L^{2}\left(\mathbb{R}^{n}\right)$, with $\eta>0$. We have $\widehat{A} \geq 0$ if and only $\operatorname{Tr}(\widehat{A} \widehat{B}) \geq 0$ for every positive trace class operator $\widehat{B} \in \mathcal{L}_{1}\left(L^{2}\left(\mathbb{R}^{n}\right)\right)$.

Proof. Since $\mathcal{L}_{1}\left(L^{2}\left(\mathbb{R}^{n}\right)\right)$ is itself an algebra the product $\widehat{A} \widehat{B}$ is indeed of trace class so the condition $\operatorname{Tr}(\widehat{A} \widehat{B}) \geq 0$ makes sense; setting $\widehat{B}=$ $\mathrm{Op}_{\eta}^{\mathrm{W}}(b)$ we have

$$
b(z)=(2 \pi \eta)^{n} \sum_{j} \beta_{j} W \psi_{j}
$$


where $\left(\beta_{j}\right) \in \ell^{1}(\mathbb{N})$ with $\beta_{j} \geq 0$ and $\psi_{j}$ an orthonormal basis for $L^{2}\left(\mathbb{R}^{n}\right)$. Observing that trace class operators are also Hilbert-Schmidt operators, we have [7, Prop. 284] since $a, b \in L^{2}\left(\mathbb{R}^{2 n}\right)$

$$
\operatorname{Tr}(\widehat{A} \widehat{B})=\int_{\mathbb{R}^{2 n}} a(z) b(z) d z
$$

and hence

$$
\operatorname{Tr}(\widehat{A} \widehat{B})=(2 \pi \eta)^{n} \sum_{j} \beta_{j} \int_{\mathbb{R}^{2 n}} a(z) W_{\eta} \psi_{j}(z) d z
$$

(the interchange of integral and series is justified by Fubini's Theorem). Assume that $\operatorname{Tr}(\widehat{A} \widehat{B}) \geq 0$. It is enough to check the positivity of $\widehat{A}$ on unit vectors $\psi$ in $L^{2}\left(\mathbb{R}^{n}\right)$. Choosing all the $\beta_{j}=0$ except $\beta_{1}$ and setting $\psi_{1}=\psi$ we have

$$
\int_{\mathbb{R}^{2 n}} a(z) W \psi(z) d z \geq 0
$$

since we can choose $\psi \in L^{2}\left(\mathbb{R}^{n}\right)$ arbitrarily, this means that we have $\widehat{A} \geq 0$. If, conversely, we have $\widehat{A} \geq 0$ then (40) holds for all $\psi_{j}$ hence $\operatorname{Tr}(\widehat{A} \widehat{B}) \geq 0$.

Let us now prove:

Theorem 16. Let $\eta>0$ and $\widehat{A}=\mathrm{Op}_{\eta}^{\mathrm{W}}(a)$ be a trace-class operator. We have $\widehat{A} \geq 0$ if and only if

$$
\int_{\mathbb{R}^{2 n}} F_{\sigma, \eta} a(z)\left(\int_{\mathbb{R}^{2 n}} e^{-\frac{i}{2 \eta} \sigma\left(z, z^{\prime}\right)} c\left(z^{\prime}-z\right) \overline{c\left(z^{\prime}\right)} d z^{\prime}\right) d z \geq 0,
$$

for all $c \in L^{2}\left(\mathbb{R}^{2 n}\right)$.

Proof. In view of Lemma [15] above we have $\widehat{A} \geq 0$ if and only if $\operatorname{Tr}(\widehat{A} \widehat{B}) \geq 0$ for all positive $\widehat{B}=\mathrm{Op}_{\eta}^{\mathrm{W}}(b) \in \mathcal{L}_{1}\left(L^{2}\left(\mathbb{R}^{n}\right)\right)$ that is (formula (39))

$$
\int_{\mathbb{R}^{2 n}} a(z) b(z) d z=(a \mid b)_{L^{2}\left(\mathbb{R}^{2 n}\right)} \geq 0 .
$$

(Recall that Weyl symbols of self-adjoint operators are real). Using Plancherel's Theorem

$$
(a \mid b)_{L^{2}\left(\mathbb{R}^{2 n}\right)}=\left(\frac{1}{2 \pi \eta}\right)^{n}\left(F_{\sigma, \eta} a \mid F_{\sigma, \eta} b\right)_{L^{2}\left(\mathbb{R}^{2 n}\right)} .
$$


Since $\widehat{B} \geq 0$ there exists $\widehat{C} \in \mathcal{L}_{2}\left(L^{2}\left(\mathbb{R}^{n}\right)\right)$ such that $\widehat{B}=\widehat{C}^{*} \widehat{C}$ and hence, setting $\widehat{C}=\mathrm{Op}_{\eta}^{\mathrm{W}}(c)$ (recall that $\widehat{C}^{*}=\mathrm{Op}_{\eta}^{\mathrm{W}}(\bar{c})$ ), by the composition formula for Weyl operators (24),

$$
\begin{aligned}
F_{\sigma, \eta} b(z) & =\left(\frac{1}{2 \pi \eta}\right)^{n} \int_{\mathbb{R}^{2 n}} e^{\frac{i}{2 \eta} \sigma\left(z, z^{\prime}\right)} F_{\sigma, \eta} \bar{c}\left(z-z^{\prime}\right) F_{\sigma, \eta} c\left(z^{\prime}\right) d z^{\prime} \\
& =\left(\frac{1}{2 \pi \eta}\right)^{n} \int_{\mathbb{R}^{2 n}} e^{\frac{i}{2 \eta} \sigma\left(z, z^{\prime}\right)} \overline{F_{\sigma, \eta} c}\left(z^{\prime}-z\right) F_{\sigma, \eta} c\left(z^{\prime}\right) d z^{\prime}
\end{aligned}
$$

Vice-versa, take any function $c \in L^{2}\left(\mathbb{R}^{2 n}\right)$ then $\widehat{C}=\mathrm{Op}_{\eta}^{\mathrm{W}}(c)$ is a Hilbert-Schmidt operator and $\widehat{B}=\widehat{C}^{*} \widehat{C}$ is a positive operator. Hence, using the fact that the operator $F_{\sigma, \eta}$ is a topological automorphism of $L^{2}\left(\mathbb{R}^{2 n}\right)$, condition (42) and (43) are equivalent to

$$
\int_{\mathbb{R}^{2 n}} F_{\sigma, \eta} a(z)\left(\int_{\mathbb{R}^{2 n}} e^{-\frac{i}{2 \eta} \sigma\left(z, z^{\prime}\right)} c\left(z^{\prime}-z\right) \overline{c\left(z^{\prime}\right)} d z^{\prime}\right) d z \geq 0,
$$

for every $c \in L^{2}\left(\mathbb{R}^{2 n}\right)$, as claimed.

4.2. Proof of the KLM condition. We are now going to prove the KLM conditions, that is Theorem 4, We will need the following classical result from linear algebra. It says that the entrywise product of two positive semidefinite matrices is also positive semidefinite.

Lemma 17 (Schur). Let $M_{(N)}=\left(M_{j k}\right)_{1 \leq j, k \leq N}$ be the Hadamard product $M_{(N)}^{\prime} \circ M_{(N)}^{\prime \prime}$ of the matrices $M_{(N)}^{\prime}=\left(M_{j k}^{\prime}\right)_{1 \leq j, k \leq N}$ and $M_{(N)}^{\prime \prime}=$ $\left(M_{j k}^{\prime \prime}\right)_{1 \leq j, k \leq N}: M_{(N)}=\left(M_{j k}^{\prime} M_{j k}^{\prime \prime}\right)_{1 \leq j, k \leq N}$. If $M_{(N)}^{\prime}$ and $M_{(N)}^{\prime \prime}$ are positive semidefinite then so is $M_{(N)}$.

Proof. See for instance Bapat [1.

We have now all the instruments to prove the KLM conditions.

Proof of Theorem 4. Let us first show that the conditions (i)-(ii) are necessary. Assume that $\widehat{A} \geq 0$. Since $a \in L^{1}\left(\mathbb{R}^{2 n}\right)$, the RiemannLebesgue Lemma gives that $a_{\diamond}$ is continuous. In view of Lemma 10 and formula (36) in Proposition 11 we have

$$
a=(2 \pi \eta)^{n} \sum_{j} \alpha_{j} W_{\eta} \psi_{j}
$$

for an orthonormal basis $\left\{\psi_{j}\right\}$ in $L^{2}\left(\mathbb{R}^{n}\right)$, the coefficients $\alpha_{j}$ being $\geq 0$ and in $\ell^{1}(\mathbb{N})$. It is thus sufficient to show that the Wigner transform $W_{\eta} \psi$ of an arbitrary $\psi \in L^{2}\left(\mathbb{R}^{n}\right)$ is of $\eta$-positive type. This amounts 
to show that for all $\left(z_{1}, \ldots, z_{N}\right) \in\left(\mathbb{R}^{2 n}\right)^{N}$ and all $\left(\zeta_{1}, \ldots, \zeta_{N}\right) \in \mathbb{C}^{N}$ we have

$$
I_{N}(\psi)=\sum_{1 \leq j, k \leq N} \zeta_{j} \overline{\zeta_{k}} e^{-\frac{i}{2 \eta} \sigma\left(z_{j}, z_{k}\right)} F_{\sigma, \eta} W_{\eta} \psi\left(z_{j}-z_{k}\right) \geq 0
$$

for every complex vector $\left(\zeta_{1}, \ldots, \zeta_{N}\right) \in \mathbb{C}^{N}$ and every sequence $\left(z_{1}, \ldots, z_{N}\right) \in$ $\left(\mathbb{R}^{2 n}\right)^{N}$. Since the $\eta$-Wigner distribution $W_{\eta} \psi$ and the $\eta$-ambiguity function are obtained from each other by the symplectic $\eta$-Fourier transform $F_{\sigma, \eta}$ we have

$$
I_{N}(\psi)=\sum_{1 \leq j, k \leq N} \zeta_{j} \overline{\zeta_{k}} e^{-\frac{i}{2 \eta} \sigma\left(z_{j}, z_{k}\right)} \operatorname{Amb}_{\eta} \psi\left(z_{j}-z_{k}\right) .
$$

Let us prove that

$$
I_{N}(\psi)=\left(\frac{1}{2 \pi \eta}\right)^{n}\left\|\sum_{1 \leq j \leq N} \zeta_{j} T_{\eta}\left(-z_{j}\right) \psi\right\|_{L^{2}}^{2}
$$

the inequality (46) will follow. Taking into account the fact that $T_{\eta}\left(-z_{k}\right)^{*}=T_{\eta}\left(z_{k}\right)$ and using the familiar relation [5, 6, 6]

$$
T_{\eta}\left(z_{k}-z_{j}\right)=e^{-\frac{i}{2 \eta} \sigma\left(z_{j}, z_{k}\right)} T_{\eta}\left(z_{k}\right) T_{\eta}\left(-z_{j}\right)
$$

we have, expanding the square in the right-hand side of (47),

$$
\begin{aligned}
\left\|\sum_{1 \leq j \leq N} \zeta_{j} T_{\eta}\left(-z_{j}\right) \psi\right\|_{L^{2}}^{2} & =\sum_{1 \leq j, k \leq N} \zeta_{j} \overline{\zeta_{k}}\left(T_{\eta}\left(-z_{j}\right) \psi \mid T_{\eta}\left(-z_{k}\right) \psi\right)_{L^{2}} \\
& =\sum_{1 \leq j, k \leq N} \zeta_{j} \overline{\zeta_{k}}\left(T_{\eta}\left(z_{k}\right) T_{\eta}\left(-z_{j}\right) \psi \mid \psi\right)_{L^{2}} \\
& =\sum_{1 \leq j, k \leq N} \zeta_{j} \overline{\zeta_{k}} e^{-\frac{i}{2 \eta} \sigma\left(z_{j}, z_{k}\right)}\left(T_{\eta}\left(z_{k}-z_{j}\right) \psi \mid \psi\right)_{L^{2}} \\
& =(2 \pi \eta)^{n} \sum_{1 \leq j, k \leq N} \zeta_{j} \overline{\zeta_{k}} e^{-\frac{i}{2 \eta} \sigma\left(z_{j}, z_{k}\right)} \mathrm{Amb}_{\eta} \psi\left(z_{j}-z_{k}\right)
\end{aligned}
$$

proving the equality (47). Let us now show that, conversely, the conditions (i) and (ii) are sufficient, i.e. that they imply that $(\widehat{A} \psi \mid \psi)_{L^{2}} \geq 0$ for all $\psi \in L^{2}\left(\mathbb{R}^{n}\right)$; equivalently (see formula (4) )

$$
\int_{\mathbb{R}^{2 n}} a(z) W_{\eta} \psi(z) d z \geq 0
$$

for $\psi \in L^{2}\left(\mathbb{R}^{n}\right)$. Let us set, as above,

$$
\Lambda_{j k}^{\prime}=e^{\frac{i}{2 \eta} \sigma\left(z_{j}, z_{k}\right)} a_{\sigma, \eta}\left(z_{j}-z_{k}\right)
$$

where $z_{j}$ and $z_{k}$ are arbitrary elements of $\mathbb{R}^{2 n}$. To say that $a_{\sigma, \eta}$ is of $\eta$-positive type means that the matrix $\Lambda^{\prime}=\left(\Lambda_{j k}^{\prime}\right)_{1 \leq j, k \leq N}$ is positive 
semidefinite; choosing $z_{k}=0$ and setting $z_{j}=z$ this means that every matrix $\left(a_{\sigma, \eta}(z)\right)_{1 \leq j, k \leq N}$ is positive semidefinite. Setting

$$
\begin{aligned}
\Gamma_{j k} & =e^{\frac{i}{2 \eta} \sigma\left(z_{j}, z_{k}\right)} F_{\sigma, \eta} W_{\eta} \psi\left(z_{j}-z_{k}\right) \\
& =e^{\frac{i}{2 \eta} \sigma\left(z_{j}, z_{k}\right)} \mathrm{Amb}_{\eta} \psi\left(z_{j}-z_{k}\right)
\end{aligned}
$$

the matrix $\Gamma_{(N)}=\left(\Gamma_{j k}\right)_{1 \leq j, k \leq N}$ is positive semidefinite. Let us now write

$$
M_{j k}=\operatorname{Amb}_{\eta} \psi\left(z_{j}-z_{k}\right) a_{\sigma, \eta}\left(z_{j}-z_{k}\right)
$$

we claim that the matrix $M_{(N)}=\left(M_{j k}\right)_{1 \leq j, k \leq N}$ is positive semidefinite. In fact, $M$ is the Hadamard product of the positive semidefinite matrices $M_{(N)}^{\prime}=\left(M_{j k}^{\prime}\right)_{1 \leq j, k \leq N}$ and $M_{(N)}^{\prime \prime}=\left(M_{j k}^{\prime \prime}\right)_{1 \leq j, k \leq N}$ where

$$
\begin{aligned}
& M_{j k}^{\prime}=e^{\frac{i}{2 \eta} \sigma\left(z_{j}, z_{k}\right)} \operatorname{Amb}_{\eta} \psi\left(z_{j}-z_{k}\right) \\
& M_{j k}^{\prime \prime}=e^{-\frac{i}{2 \eta} \sigma\left(z_{j}, z_{k}\right)} a_{\sigma, \eta}\left(z_{j}-z_{k}\right)
\end{aligned}
$$

and Lemma[17implies that $M_{(N)}$ is also positive semidefinite. It follows from Bochner's theorem that the function $b$ defined by

$$
b_{\sigma, \eta}(z)=\operatorname{Amb}_{\eta} \psi(z) a_{\sigma, \eta}(-z)
$$

is a probability density hence $b(z) \geq 0$ for all $z \in \mathbb{R}^{2 n}$. Integrating this equality we get, using the Plancherel formula for the symplectic $\eta$-Fourier transform,

$$
\begin{aligned}
(2 \pi \eta)^{n} b(0) & =\int_{\mathbb{R}^{2 n}} \operatorname{Amb}_{\eta} \psi(z) a_{\sigma, \eta}(-z) d z \\
& =\int_{\mathbb{R}^{2 n}} W_{\eta} \psi(z) a(z) d z
\end{aligned}
$$

hence the inequality (49) since $b(0) \geq 0$.

4.3. The Gaussian case. Let $\Sigma$ be a positive symmetric (real) $2 n \times 2 n$ matrix and consider the Gaussian

$$
\rho(z)=(2 \pi)^{-n} \sqrt{\operatorname{det} \Sigma^{-1}} e^{-\frac{1}{2} \Sigma^{-1} z^{2}} .
$$

Let us find for which values of $\eta$ the function $\rho$ is the $\eta$-Wigner distribution of a density operator. Narcowich [18] was the first to address this question using techniques from harmonic analysis using the approach in Kastler's paper [13] we give here a new and simpler proof using the multidimensional Hardy's uncertainty principle, which we state in the following form: 
Lemma 18. Let $A$ and $B$ be two real positive definite matrices and $\psi \in L^{2}\left(\mathbb{R}^{n}\right), \psi \neq 0$. Assume that

$$
|\psi(x)| \leq C e^{-\frac{1}{2} A x^{2}} \text { and }\left|F_{\eta} \psi(p)\right| \leq C e^{-\frac{1}{2} B p^{2}}
$$

for a constant $C>0$. Then:

(i) The eigenvalues $\lambda_{j}, j=1, \ldots, n$, of the matrix $A B$ are all $\leq 1 / \eta^{2}$;

(ii) If $\lambda_{j}=1 / \eta^{2}$ for all $j$, then $\psi(x)=k e^{-\frac{1}{2} A x^{2}}$ for some constant $k$.

Proof. See de Gosson and Luef [9], de Gosson [7]. The $\eta$-Fourier transform $F_{\eta} \psi$ in the second inequality (52) is given by (27).

We will also need the two following lemmas; the first is a positivity result.

Lemma 19. If $R$ is a symmetric positive semidefinite $2 n \times 2 n$ matrix, then

$$
P_{(N)}=\left(R z_{j} \cdot z_{k}\right)_{1 \leq j, k \leq N}
$$

is a symmetric positive semidefinite $N \times N$ matrix for all $z_{1}, \ldots, z_{N} \in$ $\mathbb{R}^{2 n}$.

Proof. There exists a matrix $L$ such that $R=L^{*} L$ (Cholesky decomposition). Denoting by $\left\langle z \mid z^{\prime}\right\rangle=z \cdot \overline{z^{\prime}}$ the inner product on $\mathbb{C}^{2 n}$ we have, since the $z_{j}$ are real vectors,

$$
L^{*} z_{j} \cdot z_{k}=\left\langle L^{*} z_{j} \mid z_{k}\right\rangle=\left\langle z_{j} \mid L z_{k}\right\rangle=z_{j} \cdot \overline{L z_{k}}
$$

hence $R z_{j} \cdot z_{k}=L z_{j} \cdot \overline{L z_{k}}$. It follows that for all complex $\zeta_{j}$ we have

$$
\sum_{1 \leq j, k \leq N} \zeta_{j} \overline{\zeta_{k}} R z_{j} \cdot z_{k}=\sum_{1 \leq j \leq N} \zeta_{j} L z_{j} \overline{\left(\sum_{1 \leq j \leq N} \zeta_{j} L z_{j}\right)} \geq 0
$$

hence our claim.

The second lemma is a well-known diagonalization result (Williamson's symplectic diagonalization theorem [5, 6]):

Lemma 20. Let $\Sigma$ be a symmetric positive definite real $2 n \times 2 n$ matrix. There exists $S \in \operatorname{Sp}(n)$ such that $\Sigma=S^{T} D S$ where

$$
D=\left(\begin{array}{cc}
\Lambda & 0 \\
0 & \Lambda
\end{array}\right)
$$

with $\Lambda=\operatorname{diag}\left(\lambda_{1}, \ldots, \lambda_{n}\right)$, the positive numbers $\lambda_{j}$ being the symplectic eigenvalues of $\Sigma$ (that is, $\pm i \lambda_{1}, \ldots, \pm i \lambda_{n}$ are the eigenvalues of $J \Sigma \backsim$ $\left.\Sigma^{1 / 2} J \Sigma^{1 / 2}\right)$.

Proof. See for instance [5, 6, 7]. 
We now have the tools needed to give a complete characterization of Gaussian $\eta$-Wigner distributions:

Proposition 21. Let $\eta \in R \backslash\{0\}$. The Gaussian function (51) is the $\eta$-Wigner transform of a positive trace class operator if and only if

$$
|\eta| \leq 2 \lambda_{\min }
$$

where $\lambda_{\min }$ is the smallest symplectic eigenvalue of $\Sigma$; equivalently the self-adjoint matrix $\Sigma+i \eta J$ is positive semidefinite:

$$
\Sigma+\frac{i \eta}{2} J \geq 0
$$

Proof. Let us first show that the conditions (154) and (155) are equivalent. Let $\Sigma=S^{T} D S$ be a symplectic diagonalization of $\Sigma$ (Lemma 201). Since $S^{T} J S=J$ condition (55) is equivalent to $D+\frac{i \eta}{2} J \geq 0$. Let $z=(x, p)$ be an eigenvector of $D+\frac{i \eta}{2} J$; the corresponding eigenvalue $\lambda$ is real and $\geq 0$. The characteristic polynomial of $D+\frac{i \eta}{2} J$ is

$$
P(\lambda)=\operatorname{det}\left[(\Lambda-\lambda I)^{2}-\frac{\eta^{2}}{4} I\right]=P_{1}(\lambda) \cdots P_{n}(\lambda)
$$

where

$$
P_{j}(\lambda)=\left(\lambda_{j}-\lambda\right)^{2}-\frac{\eta^{2}}{4}
$$

hence the eigenvalues $\lambda$ of $D+\frac{i \eta}{2} J$ are the numbers $\lambda=\lambda_{j} \pm \frac{1}{2} \eta$; since $\lambda \geq 0$ the condition $D+\frac{i \eta}{2} J \geq 0$ is equivalent to $\lambda_{j} \geq \sup \left\{ \pm \frac{1}{2} \eta\right\}=\frac{1}{2}|\eta|$ for all $j$, which is the condition (54). Let us now show that the condition (54) is necessary for the function

$$
\rho(z)=(2 \pi)^{-n} \sqrt{\operatorname{det} \Sigma^{-1}} e^{-\frac{1}{2} \Sigma^{-1} z^{2}}
$$

to be $\eta$-Wigner transform of a positive trace class operator. Let $\widehat{\rho}=$ $(2 \pi \eta)^{n} \mathrm{Op}_{\eta}^{\mathrm{W}}(\rho)$ and set $a(z)=(2 \pi \eta)^{n} \rho(z)$. Let $\widehat{S} \in \operatorname{Mp}(n)$; the operator $\widehat{\rho}$ is of trace class if only if $\widehat{S} \widehat{\rho} \widehat{S}^{-1}$ is, in which case $\operatorname{Tr}(\widehat{\rho})=$ $\operatorname{Tr}\left(\widehat{S} \widehat{\rho} \widehat{S}^{-1}\right)$. Choose $\widehat{S}$ with projection $S \in \operatorname{Sp}(n)$ such that $\Sigma=S^{T} D S$ is a symplectic diagonalization of $\Sigma$. This choice reduces the proof to the case $\Sigma=D$, that is to

$$
\rho(z)=(2 \pi)^{-n}\left(\operatorname{det} \Lambda^{-1}\right) e^{-\frac{1}{2}\left(\Lambda^{-1} x^{2}+\Lambda^{-1} p^{2}\right)} .
$$

Suppose now that $\widehat{\rho}$ is of trace class; then there exist an orthonormal basis of functions $\psi_{j} \in L^{2}\left(\mathbb{R}^{n}\right)(1 \leq j \leq n)$ such that

$$
\rho(z)=\sum_{j} \alpha_{j} W_{\eta} \psi_{j}(z)
$$


where the $\alpha_{j} \geq 0$ sum up to one. Integrating with respect to the $p$ and $x$ variables, respectively, the marginal conditions satisfied by the $\eta$-Wigner transform and formula (57) imply that we have

$$
\begin{aligned}
\sum_{j} \alpha_{j}\left|\psi_{j}(x)\right|^{2} & =(2 \pi)^{-n / 2}(\operatorname{det} \Lambda)^{1 / 2} e^{-\frac{1}{2} \Lambda^{-1} x^{2}} \\
\sum_{j} \alpha_{j}\left|F_{\eta} \psi_{j}(p)\right|^{2} & =(2 \pi)^{-n / 2}(\operatorname{det} \Lambda)^{1 / 2} e^{-\frac{1}{2} \Lambda^{-1} p^{2}} .
\end{aligned}
$$

In particular, since $\alpha_{j} \geq 0$ for every $j=1,2, \ldots, n, \ldots$,

$$
\left|\psi_{j}(x)\right| \leq C_{j} e^{-\frac{1}{4} \Lambda^{-1} x^{2}} \quad,\left|F_{\eta} \psi_{j}(p)\right| \leq C_{j} e^{-\frac{1}{4} \Lambda^{-1} p^{2}}
$$

here, if $\alpha_{j} \neq 0, C_{j}=(2 \pi)^{-n / 4}(\operatorname{det} \Lambda)^{1 / 4} / \alpha_{j}^{1 / 2}$. Applying Lemma 18 with $A=B=\frac{\eta}{2} \Lambda^{-1}$ we must have $|\eta| \leq 2 \lambda_{j}$ for all $j=1, \ldots n$, which is condition (54); this establishes the sufficiency statement. (iii) Let us finally show that, conversely, the condition (55) is sufficient. It is again no restriction to assume that $\Sigma$ is the diagonal matrix $D=\left(\begin{array}{cc}\Lambda & 0 \\ 0 & \Lambda\end{array}\right)$; the symplectic Fourier transform of $\rho$ is easily calculated and one finds that $\rho_{\diamond}(z)=e^{-\frac{1}{4} D z^{2}}$. Let $\Lambda_{(N)}=\left(\Lambda_{j k}\right)_{1 \leq j, k \leq N}$ with

$$
\Lambda_{j k}=e^{-\frac{i \eta}{2} \sigma\left(z_{j}, z_{k}\right)} \rho_{\diamond}\left(z_{j}-z_{k}\right)
$$

a simple algebraic calculation shows that we have

$$
\Lambda_{j k}=e^{-\frac{1}{4} D z_{j}^{2}} e^{\frac{1}{2}(D+i \eta J) z_{j} \cdot z_{k}} e^{-\frac{1}{4} D z_{k}^{2}}
$$

and hence

$$
\Lambda_{(N)}=\Delta_{(N)} \Gamma_{(N)} \Delta_{(N)}^{*}
$$

where $\Delta_{(N)}=\operatorname{diag}\left(e^{-\frac{1}{4} D z_{1}^{2}}, \ldots, e^{-\frac{1}{4} D z_{N}^{2}}\right)$ and $\Gamma_{(N)}=\left(\Gamma_{j k}\right)_{1 \leq j, k \leq N}$ with $\Gamma_{j k}=e^{\frac{1}{2}(D+i \eta J) z_{j} \cdot z_{k}}$. The matrix $\Lambda_{(N)}$ is thus positive semidefinite if and only if $\Gamma_{(N)}$ is, but this is the case in view of Lemma 19 .

Remark 22. Setting $2 \lambda_{\min }=\hbar$ and writing $\Sigma$ in the block-matrix form $\left(\begin{array}{cc}\Sigma_{x x} & \Sigma_{x p} \\ \Sigma_{p x} & \Sigma_{p p}\end{array}\right)$ where $\Sigma_{x x}=\left(\sigma_{x_{j}, x_{k}}\right)_{1 \leq j, k \leq n}, \Sigma_{x p}=\left(\sigma_{x_{j}, p_{k}}\right)_{1 \leq j, k \leq n}$ and so on, one shows [10] that (55) is equivalent to the generalized uncertainty relations ("Robertson-Schrödinger inequalities", see [10])

$$
\sigma_{x_{j}}^{2} \sigma_{p_{j}}^{2} \geq \sigma_{x_{j}, p_{j}}^{2}+\frac{1}{4} \hbar^{2}
$$

where, for $\leq j \leq n$, the $\sigma_{x_{j}}^{2}=\sigma_{x_{j}, x_{j}}, \sigma_{p_{j}}^{2}=\sigma_{p_{j}, p_{j}}$ are viewed as variances and the $\sigma_{x_{j}, p_{j}}^{2}$ as covariances. 


\section{The KLM Conditions in Phase SpaCe}

5.1. The main result. We now consider a Gabor (or Weyl-Heisenberg) frame $\mathcal{G}(\phi, \Lambda)$ for $L^{2}\left(\mathbb{R}^{n}\right)$, with window $\phi \in L^{2}\left(\mathbb{R}^{n}\right)$ and lattice $\Lambda \in \mathbb{R}^{n}$ (cf. (13) ). Time-frequency analysis and the Wigner formalism are the main ingredients for proving our main result.

Proof of Theorem [6. (i) Since $\mathcal{G}(\phi, \Lambda)$ is a Gabor frame, we can write

$$
\psi=\sum_{\lambda \in \Lambda} c_{\lambda} T(\lambda) \phi
$$

for some $\left(c_{\lambda}\right) \in \ell^{2}(\Lambda)$. Let us prove that

$$
\int_{\mathbb{R}^{2 n}} a(z) W_{\eta} \psi(z) d z=\sum_{\lambda, \mu \in \Lambda} c_{\lambda} \overline{c_{\mu}} e^{-\frac{i}{2 \eta} \sigma(\lambda, \mu)} a_{\lambda, \mu} .
$$

In view of the sesquilinearity of the cross-Wigner transform and its continuity as a map $L^{2}\left(\mathbb{R}^{n}\right) \times L^{2}\left(\mathbb{R}^{n}\right) \rightarrow L^{2}\left(\mathbb{R}^{2 n}\right)$ we have

$$
W_{\eta}\left(\sum_{\lambda \in \Lambda} c_{\lambda} T(\lambda) \phi\right)=\sum_{\lambda \in \Lambda} c_{\lambda} \overline{c_{\mu}} W_{\eta}(T(\lambda) \phi, T(\mu) \phi) .
$$

Using the relation (formula (9.23) in [7])

$$
W_{\eta}(T(\lambda) \phi, T(\mu) \phi)=e^{-\frac{i}{2 \eta} \sigma(\lambda, \mu)} e^{-\frac{i}{\eta} \sigma(z, \lambda-\mu)} W_{\eta} \phi\left(z-\frac{1}{2}(\lambda+\mu)\right)
$$

we obtain

$$
\begin{aligned}
\int_{\mathbb{R}^{2 n}} a & (z) W_{\eta} \psi(z) d z \\
& =c_{\lambda} \overline{c_{\mu}} e^{-\frac{i}{2 \eta} \sigma(\lambda, \mu)} \int_{\mathbb{R}^{2 n}} a(z) e^{-\frac{i}{\eta} \sigma(z, \lambda-\mu)} W_{\eta} \phi\left(z-\frac{1}{2}(\lambda+\mu)\right) d z \\
& =\sum_{\lambda, \mu \in \Lambda} c_{\lambda} \overline{c_{\mu}} e^{-\frac{i}{2 \eta} \sigma(\lambda, \mu)} a_{\lambda, \mu} .
\end{aligned}
$$

Suppose now $\widehat{A}_{\eta} \geq 0$, that is

$$
\int_{\mathbb{R}^{2 n}} a(z) W_{\eta} \psi(z) d z \geq 0
$$

for every $\psi \in L^{2}\left(\mathbb{R}^{n}\right)$. For any given sequence $\left(c_{\lambda}\right)_{\lambda \in \Lambda}$ with $c_{\lambda}=0$ for $|\lambda|>N$ we take $\psi$ as in (59) and apply (60); we obtain that the finite matrix in (15) is positive semidefinite.

Assume conversely that the matrix in (15) is positive semidefinite for every $N$; then the right-hand side of (60) is nonnegative, whenever the series converges (unconditionally). Now, every $\psi \in L^{2}\left(\mathbb{R}^{n}\right)$ has a Gabor expansion as in (59), with $c_{\lambda}=(\psi \mid T(\lambda) \gamma)_{L^{2}}$ in $\ell^{2}(\Lambda)$, for some dual window $\gamma \in L^{2}\left(\mathbb{R}^{n}\right)$. Hence from (60) and (61) we deduce $\widehat{A} \geq 0$.

(ii) The desired result follows from the following calculation: we have

$$
M_{\lambda, \mu}^{\prime}=W_{\eta}\left(a,\left(W_{\eta} \phi\right)^{\vee}\right)\left(\frac{1}{4}(\lambda+\mu), \frac{1}{2} J(\mu-\lambda)\right)
$$


that is, by definition of the Wigner transform,

$$
\begin{aligned}
M_{\lambda, \mu}^{\prime}=\left(\frac{1}{2 \pi \eta}\right)^{2 n} \int_{\mathbb{R}^{2 n}} e^{-\frac{i}{2 \eta} \sigma(\mu-\lambda, u)} a\left(\frac{1}{4}(\lambda\right. & \left.+\mu)+\frac{1}{2} u\right) \\
& \times W_{\eta} \phi\left(-\frac{1}{4}(\lambda+\mu)+\frac{1}{2} u\right) d u ;
\end{aligned}
$$

setting $z=\frac{1}{4}(\lambda+\mu)+\frac{1}{2} u$ we have $u=2 z-\frac{1}{2}(\lambda+\mu)$ and hence

$$
M_{\lambda, \mu}^{\prime}=\left(\frac{1}{2 \pi \eta}\right)^{2 n} 2^{n} \int_{\mathbb{R}^{2 n}} e^{-\frac{i}{2 \eta} \sigma\left(\mu-\lambda, 2 z-\frac{1}{2}(\lambda+\mu)\right)} a(z) W_{\eta} \phi\left(z-\frac{1}{2}(\lambda+\mu)\right) d z .
$$

Using the bilinearity and antisymmetry of the symplectic form $\sigma$ we have

$$
\sigma\left(\mu-\lambda, 2 z-\frac{1}{2}(\lambda+\mu)\right)=2 \sigma(z, \lambda-\mu)+\sigma(\lambda, \mu)
$$

so that

$$
M_{\lambda, \mu}^{\prime}=\left(\frac{1}{2 \pi \eta}\right)^{2 n} 2^{n} e^{-\frac{i}{2 \eta} \sigma(\lambda, \mu)} \int_{\mathbb{R}^{2 n}} e^{-\frac{i}{\eta} \sigma(z, \lambda-\mu)} a(z) W_{\eta} \phi\left(z-\frac{1}{2}(\lambda+\mu)\right) d z
$$

that is $M_{\lambda, \mu}^{\prime}=2^{n}(2 \pi \eta)^{-2 n} M_{\lambda, \mu}$, hence our claim.

Remark 23. Let us observe that Theorem [6 extends to other classes of symbols, essentially with the same proof. For example the results hold for $a \in M^{\infty, 1}\left(\mathbb{R}^{2 n}\right)$ (the Sjöstrand class) if the window $\phi$ belongs to $M^{1}\left(\mathbb{R}^{n}\right)$. Other choices are certainly possible.

5.2. The connection with the KLM conditions. In what follows we prove Theorem 7, which shows that the KLM conditions can be recaptured by an averaging procedure from the conditions in Theorem 6.

Proof of Theorem 7 . Let us first observe that we can write

$$
M_{\lambda, \mu}^{(K L M)}=(2 \pi \eta)^{-n} e^{-\frac{i}{2 \eta} \sigma(\lambda, \mu)} V_{\Phi} a\left(\frac{1}{2}(\lambda+\mu), J(\mu-\lambda)\right)
$$

where $\Phi(z)=1$ for all $z \in \mathbb{R}^{2 n}$. Now we have

$$
W \phi_{\nu}(z)=\left(\frac{1}{\pi \eta}\right)^{n} e^{-\frac{1}{\eta}|z-\nu|^{2}}
$$

and therefore

$$
\int_{\mathbb{R}^{2 n}} W \phi_{\nu}(z) d \nu=\left(\frac{1}{\pi \eta}\right)^{n} \int_{\mathbb{R}^{2 n}} e^{-\frac{1}{\eta}|z-\nu|^{2}} d \nu=1=\Phi(z) \quad \forall z \in \mathbb{R}^{2 n} .
$$

Hence (19) follows by exchanging the integral with respect to $\nu$ in (19) with the integral in the definition of the STFT in (18). Fubini's theorem can be applied because the function

$$
a(z) W \phi_{\nu}(z-\zeta)
$$

belongs to $L^{1}\left(\mathbb{R}^{2 n} \times \mathbb{R}^{2 n}\right)$ with respect to $z, \nu$, for every fixed $\zeta \in$ $\mathbb{R}^{2 n}$. 
Corollary 24. Suppose $a \in L^{1}\left(\mathbb{R}^{n}\right) \cap L^{2}\left(\mathbb{R}^{n}\right)$. With the notation in Theorem 7 , suppose that $\mathcal{G}\left(\phi_{0}, \Lambda\right)$ is a Gabor frame for $L^{2}\left(\mathbb{R}^{n}\right)$. If the matrix $\left(M_{\lambda, \mu}^{\phi_{0}}\right)_{\lambda, \mu \in \Lambda,|\lambda|,|\mu| \leq N}$ is positive semidefinite for every $N$, then so is the matrix $\left(M_{\lambda, \mu}^{(K L M)}\right)_{\lambda, \mu \in \Lambda,|\lambda|,|\mu| \leq N}$.

Proof. Observing that $\mathcal{G}\left(\phi_{\nu}, \Lambda\right)$ is also a Gabor frame for every $\nu \in$ $\mathbb{R}^{2 n}$, it follows from the assumptions and Theorem 6 that the matrices $\left(M_{\lambda, \mu}^{\phi_{\nu}}\right)_{\lambda, \mu \in \Lambda,|\lambda|,|\mu| \leq N}$ are positive semidefinite for all $\nu \in \mathbb{R}^{2 n}$. The result therefore follows from (19).

Corollary 25. Suppose $a \in L^{1}\left(\mathbb{R}^{n}\right) \cap L^{2}\left(\mathbb{R}^{n}\right)$ and $\widehat{A}_{\eta}=\mathrm{Op}_{\eta}^{\mathrm{W}}(a) \geq 0$. Then, with the notation in Theorem $\square$, for every finite subset $S \subset \mathbb{R}^{2 n}$ the matrix $\left(M_{\lambda, \mu}^{(K L M)}\right)_{\lambda, \mu \in S}$ is positive semidefinite (that is, the KLM conditions hold).

Proof. Since $\mathcal{G}\left(\phi_{0}, \Lambda\right)$ is a frame for $L^{2}\left(\mathbb{R}^{n}\right)$ for every sufficiently dense lattice $\Lambda$, as a consequence of Corollary 24 the matrix $\left(M_{\lambda, \mu}^{(K L M)}\right)_{\lambda, \mu \in \Lambda,|\lambda|,|\mu| \leq N}$ is positive semidefinite for all such lattices $\Lambda$ and every integer $N$. By restricting the matrix to subspaces we see that the submatrices $\left(M_{\lambda, \mu}^{(K L M)}\right)_{\lambda, \mu \in S}$ are positive semidefinite for every finite subset $S \subset \Lambda$. Since $a \in L^{1}\left(\mathbb{R}^{n}\right)$ the symplectic Fourier transform $a_{\sigma, \eta}$ is continuous and therefore the same holds for every finite subset $S \subset \mathbb{R}^{2 n}$.

5.3. Almost positivity. We now address the following question: suppose that $\mathcal{G}(\phi, \Lambda)$ is a Gabor frame for $L^{2}\left(\mathbb{R}^{n}\right)$ and assume that the matrix $\left(M_{\lambda, \mu}\right)_{\lambda, \mu \in \Lambda,|\lambda|,|\mu| \leq N}$ in (15), (14) is positive semidefinite for a fixed $N$. What can we say about the positivity of the operator $\widehat{A}_{\eta}$ ? Under suitable decay condition on the symbol $a$ it turns out that $\widehat{A}_{\eta}$ is "almost positive" in the following sense.

Let $\mathcal{G}(\phi, \Lambda)$ be a Gabor frame in $L^{2}\left(\mathbb{R}^{n}\right)$, with $\phi \in \mathcal{S}\left(\mathbb{R}^{n}\right)$.

Theorem 26. Let $a \in M_{v_{s}}^{\infty, 1}\left(\mathbb{R}^{2 n}\right)$ be real valued and $s \geq 0$; we use the notation $v_{s}(\zeta)=\langle\zeta\rangle^{s}$ for $\zeta \in \mathbb{R}^{4 n}$. Suppose that the matrix

$$
\left(M_{\lambda, \mu}\right)_{\lambda, \mu \in \Lambda,|\lambda|,|\mu| \leq N}
$$

in (15) is positive semidefinite for some integer $N$. Then there exists a constant $C>0$ independent of $N$ such that

$$
\left(\widehat{A}_{\eta} \psi \mid \psi\right)_{L^{2}} \geq-C N^{-s}\|\psi\|_{L^{2}}^{2}
$$

for all $\psi \in L^{2}\left(\mathbb{R}^{n}\right)$.

Proof. Let $\psi \in L^{2}\left(\mathbb{R}^{n}\right)$, and write its Gabor frame expansion as

$$
\psi=\sum_{\lambda \in \Lambda,|\lambda| \leq N} c_{\lambda} T(\lambda) \phi+\sum_{\lambda \in \Lambda,|\lambda|>N} c_{\lambda} T(\lambda) \phi ;
$$


denoting the sums in the right-hand side by, respectively, $\psi^{\prime}$ and $\psi^{\prime \prime}$ we get

$\left(\widehat{A}_{\eta} \psi \mid \psi\right)_{L^{2}}=\left(\widehat{A}_{\eta} \psi^{\prime} \mid \psi^{\prime}\right)_{L^{2}}+\left(\widehat{A}_{\eta} \psi^{\prime} \mid \psi^{\prime \prime}\right)_{L^{2}}+\left(\widehat{A}_{\eta} \psi^{\prime \prime} \mid \psi^{\prime}\right)_{L^{2}}+\left(\widehat{A}_{\eta} \psi^{\prime \prime} \mid \psi^{\prime \prime}\right)_{L^{2}}$

We have, by (60) and the positivity assumption

$$
\left(\widehat{A}_{\eta} \psi^{\prime} \mid \psi^{\prime}\right)_{L^{2}}=\sum_{\lambda, \mu \in \Lambda,|\lambda|,|\mu| \leq N} c_{\lambda} \overline{c_{\mu}} M_{\lambda, \mu} \geq 0
$$

hence it is sufficient to show that

$$
\left|\left(\widehat{A}_{\eta} \psi^{\prime} \mid \psi^{\prime \prime}\right)_{L^{2}}\right| \leq C N^{-s}|| \psi \|_{L^{2}}^{2}
$$

and similar inequalities for the other terms. Now

$$
\left|\left(\widehat{A}_{\eta} \psi^{\prime} \mid \psi^{\prime \prime}\right)_{L^{2}}\right|=\left|\left(\psi^{\prime} \mid \widehat{A}_{\eta} \psi^{\prime \prime}\right)_{L^{2}}\right| \leq\left\|\psi^{\prime}\right\|_{L^{2}}|| \widehat{A}_{\eta} \psi^{\prime \prime} \|_{L^{2}}
$$

Observe that the function $\psi^{\prime \prime}$ has a Gabor expansion with coefficients $c_{\lambda}=0$ for $|\lambda| \leq N$. By the frame property and the same computation as in the proof of (60) we have

$$
\begin{aligned}
\left\|\widehat{A}_{\eta} \psi^{\prime \prime}\right\|_{L^{2}} & \asymp\left\|\left(\widehat{A}_{\eta} \psi^{\prime \prime} \mid T(\mu) \phi\right)_{L^{2}}\right\|_{\ell^{2}} \\
& =\left\|\sum_{\lambda \in \Lambda,|\lambda|>N} M_{\lambda, \mu} c_{\lambda}\right\|_{\ell^{2}} .
\end{aligned}
$$

Observe that (14) can be rewritten in terms of the short-time Fourier transform $(\mathrm{STFT})$ on $\mathbb{R}^{2 n}$ as

$$
a_{\lambda, \mu}=V_{W_{\eta} \phi} a\left(\frac{1}{2}(\lambda+\mu), J(\mu-\lambda)\right) .
$$

Now, from (15) and (64) we have

$$
\left|M_{\lambda, \mu}\right|=\left|V_{W_{\eta} \phi} a\left(\frac{1}{2}(\lambda+\mu), J(\mu-\lambda)\right)\right| .
$$

In view of the assumption $a \in M_{v_{s}}^{\infty, 1}$ we have, by [12, Theorem 12.2.1], that

$$
\sum_{\nu \in \Lambda^{\prime}} \sup _{z \in \mathbb{R}^{2 n}}(1+|z|+|\nu|)^{s}\left|V_{W_{\eta} \phi} a(z, \nu)\right|<\infty
$$

for every lattice $\Lambda^{\prime} \subset \mathbb{R}^{2 n}$. Now we apply this formula with $\Lambda^{\prime}=J(\Lambda)$; using

$$
1+|\lambda|+|\mu| \asymp 1+\frac{1}{2}|\lambda+\mu|+|J(\mu-\lambda)|
$$

we obtain, for $|\lambda|>N$

$$
\begin{aligned}
N^{s}\left|M_{\lambda, \mu}\right| & \leq C\left(1+\frac{1}{2}|\lambda+\mu|+|J(\mu-\lambda)|\right)^{s}\left|V_{W_{\eta} \phi} a\left(\frac{1}{2}(\lambda+\mu), J(\mu-\lambda)\right)\right| \\
& \leq H(\mu-\lambda)
\end{aligned}
$$

for some $H \in \ell^{1}(\Lambda)$. By Schur's test we can continue the above estimate as

$$
\left\|\sum_{\lambda \in \Lambda,|\lambda|>N} M_{\lambda, \mu} c_{\lambda}\right\|_{\ell^{2}} \leq C N^{-s}\left\|c_{\lambda}\right\|_{\ell^{2}}
$$


which combined with (63) gives (62), because

$$
\left\|c_{\lambda}\right\|_{\ell^{2}} \asymp\left\|\psi^{\prime \prime}\right\|_{L^{2}} \leq C^{\prime}\|\psi\|_{L^{2}}
$$

and $\left\|\psi^{\prime}\right\|_{L^{2}} \leq C^{\prime \prime}|| \psi \|_{L^{2}}$.

\section{ACKNOWLEDGEMENTS}

M. de Gosson has been funded by the Grant P27773 of the Austrian Research Foundation FWF. E. Cordero and F. Nicola were partially supported by the Gruppo Nazionale per l'Analisi Matematica, la Probabilità e le loro Applicazioni (GNAMPA) of the Istituto Nazionale di Alta Matematica (INdAM).

\section{REFERENCES}

[1] R. Bapat, Nonnegative Matrices and Applications, Cambridge University Press, 1997.

[2] P. Blanchard and E. Brüning, Mathematical Methods in Physics: Distributions, Hilbert Space Operators, Variational Methods, and Applications in Quantum Physics. Vol. 69. Birkhäuser, 2015.

[3] S. Bochner, Lectures on Fourier Integrals, Princeton University Press, Princeton, N.J., 1959.

[4] N. C. Dias, and J. N. Prata, The Narcowich-Wigner spectrum of a pure state, Rep. Math. Phys. 63(1), 43-54 (2009).

[5] G. B. Folland, Harmonic Analysis in Phase space. Annals of Mathematics studies, Princeton University Press, Princeton, N. J., 1989.

[6] M. de Gosson, Symplectic Geometry and Quantum Mechanics. Birkhäuser, Basel, series "Operator Theory: Advances and Applications" (subseries: "Advances in Partial Differential Equations"), Vol. 166, 2006.

[7] M. de Gosson, Symplectic Methods in Harmonic Analysis and in Mathematical Physics, Birkhäuser, 2011.

[8] M. de Gosson, Introduction to Born-Jordan Quantization, Springer-Verlag, series Fundamental Theories of Physics, 2016.

[9] M. de Gosson and F. Luef, Quantum states and Hardy's formulation of the uncertainty principle: a symplectic approach, Lett. Math. Phys. 80(1) (2007) 69-82.

[10] M. de Gosson and F. Luef, Symplectic Capacities and the Geometry of Uncertainty: the Irruption of Symplectic Topology in Classical and Quantum Mechanics. Phys. Reps. 484 (2009)

[11] H. G. Feichtinger, Modulation spaces on locally compact abelian groups, Technical Report, University Vienna, 1983, and also in Wavelets and Their Applications, M. Krishna, R. Radha, S. Thangavelu, editors, Allied Publishers, 99-140, 2003.

[12] K. Gröchenig, Foundations of Time-Frequency Analysis, Birkhäuser, Boston 2000.

[13] D. Kastler, The $C^{*}$-Algebras of a Free Boson Field, Commun. math. Phys. 1, 14-48 (1965).

[14] Y. Katznelson, An Introduction to Harmonic Analysis, Dover, New York, 1976. 
[15] G. Loupias and S. Miracle-Sole, $C^{*}$-Algèbres des systèmes canoniques, I, Commun. math. Phys., 2, 31-48 (1966).

[16] G. Loupias and S. Miracle-Sole, $C^{*}$-Algèbres des systèmes canoniques, II, Ann. Inst. Henri Poincaré, 6(1), 39-58 (1967).

[17] G. Mourgues, M. R. Feix, and J. C. Andrieux, Not necessary but sufficient condition for the positivity of generalized Wigner functions, J. Math. Phys, 26(10) 2554-2555 (1985).

[18] F. J. Narcowich, Conditions for the convolution of two Wigner distributions to be itself a Wigner distribution, J. Math. Phys., 29(9), 2036-2041 (1988).

[19] F. J. Narcowich, Distributions of $\eta$-positive type and applications, J. Math. Phys., 30(11), 2565-2573 (1989).

[20] F. J. Narcowich, Geometry and uncertainty, J. Math. Phys., 31(2),354-364 (1990).

[21] F. J. Narcowich and R. F. O'Connell, Necessary and sufficient conditions for a phase-space function to be a Wigner distribution, Phys, Rev. A, 34(1), 1-6 (1986).

[22] F. J. Narcowich and R. F. O'Connell, A unified approach to quantum dynamical maps and Gaussian Wigner distributions, Phys. Lett. A, 133(4) 167-170 (1988).

[23] K. R. Parthasarathy, An introduction to quantum stochastic calculus, Springer Science \& Business Media, 2012.

[24] K. R. Parthasarathy, What is a Gaussian state?, Commun. Stoch. Anal, 4(2) 143-160 (2010).

[25] K. R. Parthasarathy, and K. Schmidt, Positive definite kernels, continuous tensor products, and central limit theorems of probability theory, Lecture Notes in Mathematics, vol. 272, Springer, 1972.

[26] J. Sjöstrand. An algebra of pseudodifferential operators. Math. Res. Lett., 1(2), 185-192 (1994).

[27] M. D. Srinivas and E. Wolf, Some nonclassical features of phase-space representations of quantum mechanics, Phys. Rev. D, 11(6),1477-1485 (1975).

[28] R. Werner, Quantum harmonic analysis on phase space, J. Math. Phys., 25(5), 1404-1411 (1984).

Dipartimento di Matematica, Università di Torino, Dipartimento Di Matematica, via Carlo Alberto 10, 10123 Torino, Italy

E-mail address: elena.cordero@unito.it

University of Vienna, Faculty of Mathematics, Oskar-Morgenstern-

Platz 1 A-1090 Wien, Austria

E-mail address: maurice.de.gosson@univie.ac.at

Dipartimento di Scienze Matematiche, Politecnico di Torino, corso Duca Degli Abruzzi 24, 10129 Torino, ItAly

E-mail address: fabio.nicola@polito.it 